\title{
RELACIÓN ENTRE LA INTELIGENCIA EMOCIONAL Y LA ACTITUD HACIA LA DIVERSIDAD EN ESTUDIANTES DE PEDAGOGÍA
}

RELATIONSHIP BETWEEN EMOTIONAL INTELLIGENCE AND THE ATTITUDE TOWARDS DIVERSITY AMONG PEDAGOGY STUDENTS

\author{
Nicolás Ponce Díaz , Milenko Del Valle Tapia**, \\ Pablo Camus Galleguillos****, Gabriel Álvarez Morgado*****
}

\section{Resumen}

La posibilidad que tienen los profesores y profesoras de configurar espacios inclusivos que permitan atender a la diversidad, depende, en gran medida, de la formación recibida en las Instituciones de Educación Superior. Desde esta perspectiva, el objetivo de este estudio es describir la relación entre la Inteligencia Emocional (IE) y las Actitudes hacia la Diversidad (personal, cognitiva y cultural), presentes en estudiantes de pedagogía de dos universidades tradicionales de la región de Antofagasta, Chile. En el estudio de tipo no experimental, de corte transversal y alcance correlacional, participaron 51 estudiantes. Los datos se recogieron a través de las escalas Trait Meta-Mood Scale (TMMS-24) y Actitud Hacia la Diversidad (ACTDIV). Los resultados muestran que no existe relación entre ambas variables (rho $=0.204 ; \mathrm{p}=0,152$ ), pero sí en algunas de sus dimensiones.

* Magíster Psicología Educacional, Departamento de Educación, Universidad de Antofagasta, email: nicolas.ponce@uantof.cl

** Doctor en Psicología, Departamento de Ciencias Sociales, Universidad de Antofagasta, email: milenko.delvalle@uantof.cl

*** Doctor en Sociología, Departamento de Educación, Universidad de Antofagasta, email: pablo.camus@uantof.cl

**** Licenciado en Estadística, Observatorio de Educación, Universidad de Antofagasta, email: gabriel.alvarez.morgado@uantof.cl 
Palabras Clave: Inteligencia, enseñanza superior, formación de docentes, actitud del estudiante, diversidad.

\begin{abstract}
The possibility that teachers have to configure inclusive spaces that allow them to deal with diversity, depends to a great extent on the training received from Higher Education Institutions. From this perspective, the objective of this study is to describe the relationship between Emotional Intelligence (EI) and Attitudes towards Diversity (personal, cognitive and cultural) present in students of pedagogy from two traditional universities in the region of Antofagasta, Chile. In the non-experimental, cross-sectional and correlational-scope study, 51 students participated. Data were collected through the Trait Meta-Mood Scale (TMMS24) and Attitude Towards Diversity (ACTDIV) scales. The results show that there is no relationship between both variables ( $r h o=0.204, p=0.152$ ), but there is in some of its other dimensions.
\end{abstract}

Keywords: Student assessment, pedagogical practice in assessment, educational strategies, teacher education, vulnerable teaching institutions.

\title{
1. Introducción
}

U NO DE LOS PRINCIPALES rasgos que ha caracterizado a la Educación Superior en Chile y en América Latina durante los últimos años es el de la masificación o aumento del acceso a ella. Tradicionalmente, a la universidad accedían personas de elite con mejores desempeños y pertenecientes a grupos socioeconómicos más favorecidos (Peña, Williamson y González-Fiegehen, 2006). Hoy la educación universitaria ha perdido parte de su homogeneidad y han ingresado a ella personas de los más diversos orígenes sociales y culturales, lo que ha permitido avanzar en la configuración de espacios ricos en diversidad cultural, social y económica, por nombrar algunas (Peña et al, 2006). No obstante, dicha heterogeneidad aún no es suficiente; estudiantes y profesores no encuentran suficiente diversidad como para verse motivados a trabajar en la construcción de espacios inclusivos. 
Los estudiantes, en la mayor parte de los planteles universitarios, conviven (mayoritaria, aunque no exclusivamente) con personas parecidas entre sí, por lo que responden a un currículum y a prácticas educativas que tienden a lo homogéneo antes que a lo diverso y desarrollan así habilidades y capacidades que no siempre dan cuenta de la riqueza de las diferencias personales. Bajo este contexto, es de responsabilidad de las instituciones de Educación Superior el desarrollo de mecanismos formativos que sean coherentes con las competencias básicas a adquirir en materia de atención a la diversidad social y cultural, tanto a nivel cognitivo como a nivel emocional y actitudinal (Eddy, 2018).

Por este motivo, la presente investigación tiene como propósito profundizar en la temática de la diversidad y la Inteligencia Emocional (IE) en instituciones de Educación Superior, y se planteó como objetivo describir la relación entre la inteligencia emocional y las actitudes hacia la diversidad, que presentan estudiantes de pedagogía de la Facultad de Educación de la Universidad de Antofagasta y de la Escuela de Educación de la Universidad Católica del Norte.

La literatura especializada demuestra la importancia de las actitudes del profesorado y el desarrollo de su IE, a la hora de promover espacios inclusivos dentro de los centros educativos, como una estrategia que contribuye a que niños y niñas alcancen su máximo potencial como parte del proceso de desarrollo.

\section{Inteligencia Emocional}

Desde fines de los años 80, se ha despertado un gran interés científico por recoger información respecto de la relación que existe entre las creencias y la construcción de juicios por parte de los individuos y la influencia de dicha relación en la toma de decisiones (FernándezBerrocal y Extremera, 2005). En concordancia con este interés y para comprender cómo las emociones influyen en el pensamiento, producto del uso que se le da al afecto como fuente de información, surge una nueva línea de investigación cimentada en el concepto de Inteligencia Emocional (IE). Esta línea, desde un marco teórico-conceptual, permite profundizar en las distintas configuraciones cognitivas que realizan 
los individuos al momento de evaluar los sentimientos (Extremera y Fernández-Berrocal, 2005). En este sentido, tal como lo plantean Extremera y Fernández-Berrocal (2005); Schutte et. al (2001) y Palomera, Fernández-Berrocal y Brackett, 2008), gran parte de la expectativa puesta en el estudio de la IE está en el carácter predictivo que esta posee, respecto del funcionamiento social y personal del individuo.

La literatura plantea la existencia de tres grandes modelos que permiten categorizar la IE: el "modelo Bar-On", el "modelo de Goleman" y el "modelo de Salovey-Mayer". El primero describe una sección transversal de competencias emocionales y sociales que afectarían el comportamiento inteligente. El segundo considera a la IE como una amplia gama de competencias y habilidades que promueven el desempeño en el trabajo. Y el tercero la define como la capacidad de percibir, entender, administrar y regular las emociones, tanto a nivel personal como también de los demás (Espinoza, Sanhueza, Ramírez y Sáez, 2015).

Para los fines de esta investigación, se considerará el modelo de Salovey-Mayer, el cual, desde la especificidad de su posición, permite conceptualizar la IE a través de cuatro habilidades básicas: (1) La habilidad para percibir, valorar y expresar emociones con exactitud, (2) la habilidad para acceder y/o generar sentimientos que faciliten el pensamiento, (3) la habilidad para comprender emociones y el conocimiento emocional y (4) la habilidad para regular las emociones promoviendo un crecimiento emocional e intelectual (Mayer y Salovey, 1997, p. 10).

Desde esta mirada, Mayer y Salovey (1997) plantean el modelo de IE como un conjunto de habilidades dispuestas jerárquicamente, que van desde las más básicas -como la percepción de la emoción con exactitud (proceso psicológico básico)- hasta las más complejas como son la regulación y promoción del crecimiento emocional intelectual (proceso psicológico superior). Según el modelo, es necesario el desarrollo de habilidades básicas para promover aquellas más complejas. Dicha estructura jerárquica puede disponerse de la siguiente forma: a) Percepción, evaluación y expresión de las emociones (identificación fisiológica y cognitiva, intra e interpersonal de las emociones); b) La emoción como facilitadora del pensamiento (la emoción como estructuradora del pensamiento y del procesamiento de la información, lo 
que permite adoptar diferentes puntos de vista y múltiples perspectivas de los problemas); c) El conocimiento emocional (comprensión de emociones básicas y complejas); y d) La regulación de las emociones (manejo de las emociones a nivel intra e interpersonal) (Cabello, Ruiz-Aranda y Fernández-Berrocal, 2010).

Ahora bien, al considerar las últimas dos décadas, la afectividad y el desarrollo de las capacidades sociales y emocionales de niños y niñas en el ámbito educativo ha cobrado importancia (Palomera, Fernández-Berrocal y Brackett, 2008). Ante este contexto, se destaca la importancia que hoy tienen las distintas instituciones que forman docentes, las cuales deben apuntar al desarrollo de competencias socio-emocionales que le permitan al futuro profesor percibir, expresar, comprender y evaluar las distintas emociones y sentimientos que surgen, por una parte, del proceso enseñanza-aprendizaje y, por otra, de las distintas interacciones sociales (Bisquerra, 2011).

En este sentido, el desarrollo de competencias emocionales tanto en niños y niñas como en los futuros docentes son necesarias no solo para la prevención de problemas de salud mental, sino también para la creación de ambientes favorable de aprendizaje (Palomera et al., 2008).

Bajo este contexto, y de acuerdo con lo planteado en Cabello, RuizAranda y Fernández-Berrocal (2010), las distintas instituciones educativas deben proveer los espacios físicos, sociales y emocionales que permitan el desarrollo de una serie de herramientas con las cuales, tanto docentes como estudiantes, puedan afrontar los diversos desafíos que la vida cotidiana presenta.

Según lo anterior, es de vital importancia incluir -tanto en las instituciones que forman docentes como en las distintas escuelas- programas que permitan el desarrollo de competencias o habilidades emocionales, las cuales se configuran en un armonizador de actitudes que permiten la creación de espacios inclusivos.

\section{Actitudes: concepto, modelo comprensivo y su relevancia para la docencia}

En términos generales, y de acuerdo con lo planteado por Rodríguez 
(1991), podemos entender la actitud como una organización duradera de creencias y cogniciones en general, dotada de una carga afectiva a favor o en contra de un objeto definido, que predispone a una acción coherente con las cogniciones y afectos relativos a dicho objeto. Para hacer esta definición aún más consistente con los temas claves de este trabajo, podríamos decir que las actitudes se orientan hacia "objetos sociales", categoría en la cual podemos situar la acción educativa.

Rodríguez (1991) distingue tres componentes que conforman las actitudes, a saber: a) Componente cognoscitivo, para que exista una actitud, es necesario que exista también una representación cognoscitiva del objeto. Dicha percepción está formada por las percepciones y creencias hacia un objeto, así como por la información que tenemos sobre el mismo. En este caso se habla de modelos actitudinales de expectativa por valor, sobre todo en referencia a los estudios de Ajzen y Fishbein (1980). b) Componente afectivo, es el sentimiento en favor o en contra de un objeto social. Es el componente más característico de las actitudes. Aquí radica la diferencia principal con las creencias y las opiniones, que se caracterizan por su componente cognoscitivo. c) Componente conductual: es la tendencia a reaccionar hacia los objetos de una determinada manera. Es el componente activo de la actitud.

Para explicar la relación entre actitud y conducta, Ajzen y Fishbein (1980) han desarrollado una teoría general del comportamiento, que integra un grupo de variables que se encuentran relacionadas con la toma de decisiones a nivel conductual. La Teoría de la Acción Razonada (Ajzen y Fishbein, 1980) concibe la conducta como el resultado final de un proceso pensado, elaborado, racional y lógico. La conducta sería el eslabón final de una cadena que se inicia con la intención de (realizar) una conducta. De acuerdo con esta teoría, la conducta está determinada por la intención de llevar a cabo un determinado comportamiento y es considerada como el antecedente inmediato de la conducta. La intención está determinada a su vez por la evaluación positiva o negativa que cada sujeto hace del objeto de actitud (actitud hacia la conducta) y por la presión que ejerce el grupo o los grupos significativos para el sujeto (norma subjetiva). Pero el éxito de llevar a cabo una conducta depende no solo de una intención favorable, sino también de un nivel suficiente de control conductual, con lo que esta 
variable también puede relacionarse, al igual que la intención, de forma directa con la conducta. En este sentido, Ajzen y Fishbein (1980) afirman que la actitud final será positiva, si una persona cree que la conducta le va a permitir alcanzar objetivos gratificantes, y será negativa en caso contrario.

Bajo este contexto, la actitud definida por Fishbein, según (Reyes, 2007, p.68) es entendida como "la posición de una persona sobre una dimensión bipolar evaluativa o afectiva con respecto de un objeto acción o evento", es decir, representa el sentimiento a favor o en contra de una persona hacia un objeto estímulo externo (Ajzen y Fishbein, 1980), en que el componente emocional juega un rol importante en la evaluación cognitiva que el sujeto hace tanto del entorno como de su interior (Ramos, Piqueras, Martínez, y Oblitas, 2009). Dicha evaluación condicionaría la posición del sujeto frente al objeto.

Desde esta mirada, es relevante la formación de los futuros profesores con un modelo que les permita, por un lado, adaptarse a las diferencias del alumnado y, por otro, depositar altas expectativas en ellos. Dichas expectativas influirán directamente en el desarrollo de competencias o habilidades emocionales, no solo de quienes presentan alguna necesidad especial, sino de todo el estudiantado (Sanhueza, Granada y Bravo, 2012). Esta formación o construcción de identidades de futuros pedagogos, desde discursos inclusivos, permitirá construir actitudes favorables hacia la diversidad (Infante, 2010).

La formación de docentes con una 'actitud favorable hacia la diversidad' no es solo un imperativo moral o un mandato socialmente deseable. Es un factor que incide directamente en la calidad de los procesos formativos, que van desde las prácticas pedagógicas hasta la articulación con el contexto familiar del estudiante (Sales, Moliner, y Sanchiz, 2001).

Con todo, esta investigación plantea dos hipótesis: (1) la existencia de una relación significativa entre la inteligencia emocional y actitudes hacia la diversidad en estudiantes de pedagogía de la Facultad de Educación de la Universidad de Antofagasta y de la Escuela de Educación de la Universidad Católica del Norte. (2) La existencia de diferencias significativas en las variables anteriormente mencionadas, al comparar los resultados entre diferentes subgrupos de la muestra. 
Para tal efecto, la muestra será dividida según algunas variables de interés como sexo, carrera que cursa, entre otras.

De esta manera, los objetivos que guiaron la investigación fueron los siguientes: (1) determinar la relación entre las actitudes hacia la diversidad y la inteligencia emocional de los y las estudiantes de pedagogía de estas universidades; y (2) Comparar las actitudes hacia la diversidad y la inteligencia emocional entre diferentes subgrupos muestrales.

\section{Metodología}

\subsection{Tipo de Estudio}

Dados el propósito y las características de los constructos considerados, el abordaje metodológico plantea una investigación de enfoque cuantitativo. El diseño es de tipo no experimental, corte transversal y alcance correlacional, ya que tiene por objetivo describir la relación entre las variables -inteligencia emocional y actitud hacia la diversidad-, en un momento determinado y sin manipular deliberadamente las variables.

\subsection{Instrumento de recolección de datos}

Para la recolección de datos, se aplicaron dos escalas tipo Likert y un cuestionario enfocado en caracterizar socio-demográficamente a los estudiantes.

La primera escala tipo Likert, que mide actitud hacia la diversidad, es una adaptación de la escala ACTDIV propuesta por Solórzano (2013), la cual fue sometida a un proceso de validación cruzada mediante un análisis factorial confirmatorio y midiendo su fiabilidad a través del estadístico Alpha de Cronbach $(\alpha=0,817$ y $\alpha=0,802$, en cada muestra respectivamente).

Esta escala mide la actitud hacia la diversidad en sus componentes cognitivo, conductual y emocional, mediante un instrumento compuesto por 20 ítems dispuestos bajo tres sub-constructos, a saber:

a) Diversidad Personal: aquellas características que hacen a cada persona un ser único e irrepetible por sus características físicas y 
de derecho. Se pueden citar como indicadores: la edad, el sexo, el estado civil y la preferencia sexual. b) Diversidad Cultural: corresponde a los rasgos que caracterizan y definen a cada cultura y que le pertenece a cada persona por ser parte de ella, se pueden distinguir la lengua, la religión, la raza, la etnia, las prácticas sociales y políticas. c) Diversidad Cognitiva: comprende las diversas formas en que las personas se acercan a los objetos de aprendizaje y construyen e interiorizan el conocimiento, se consideran en este aspecto los estilos de aprendizaje, las habilidades personales y las experiencias previas que definen los significados propios de los objetos de aprendizaje (Solórzano, 2013, p.7).

Cabe destacar que, y considerando a la "diversidad" como un concepto casi infinito, los sub-constructos expuestos con anterioridad corresponden a una construcción teórica parcial que la autora realiza. Para Solórzano (2013) dichos componentes "visitan y conviven en el aula en el diario quehacer del proceso de enseñanza y aprendizaje" (p.7).

Cada ítem posee una escala tipo Likert con opciones de respuestas de 5 niveles, en que el mínimo es cero ("totalmente en desacuerdo") y el máximo es 4 ("totalmente de acuerdo"). Las aseveraciones en sentido inverso fueron recodificadas para los análisis finales.

La segunda escala es la Trait-Meta Mood Scale-24 propuesta por Salovey, Mayer, Goldman, Turvey y Palfai (1995) y validada en la población chilena por Espinoza, Sanhueza, Ramírez y Sáez, (2015) en una muestra compuesta por 349 estudiantes de Educación Superior. La fiabilidad de este instrumento fue medido a través del estadístico alfa de Cronbach $(\alpha=0,95)$.

La escala está compuesta por 24 ítems, los cuales permiten describir la capacidad que tienen los sujetos para percibir, entender, administrar y regular las emociones intra e interpersonales, bajo tres dimensiones, a saber: a) Percepción Emocional: reconocimiento e identificación de lo que producen las propias emociones, a las que se puede asignar una etiqueta verbal. b) Comprensión Emocional: Considera la complejidad de los cambios emocionales, integrando elementos cognitivos y emocionales. c) Regulación Emocional: dirección y manejo eficaz de las emociones (positivas y negativas). Cada ítem posee una escala tipo 
Likert con opciones de respuesta de 5 niveles, en que el mínimo es cero ("Nada de acuerdo") y el máximo es 4 ("Totalmente de acuerdo").

Cabe destacar que ambos instrumentos fueron utilizados con la autorización de sus autores.

Finalmente, las variables sociodemográficas indagadas dan cuenta del nivel de enseñanza de la carrera que estudia, sexo del estudiante, nivel educativo de los padres, dependencia administrativa del colegio de procedencia y participación en actividades que promueven la inclusión.

\subsection{Participantes}

La población objetivo está conformada por los estudiantes de cuarto año de las carreras de pedagogía pertenecientes a las Universidades Tradicionales de la región de Antofagasta, Chile.

Para el estudio, se planteó realizar un censo sobre la población. Los estudiantes fueron encuestados en salas de clases de asignaturas de cuarto año. Se recopilaron un total de 86 encuestas, lo que refleja una tasa de respuesta del 86\%. No hay razón aparente para sospechar que las variables de estudio estén relacionadas con la ausencia de respuesta, por lo que los estudiantes faltantes serán tratados como «faltantes completamente al azar» (en inglés, Missing Completely At Random, MCAR). De esta manera, los cuestionarios recolectados serán tratados como una muestra aleatoria de la población objetivo (Lohr, 2000).

De las 86 encuestas, fueron eliminadas 9 por no haber contestado las escalas completamente, 13 por no cumplir con los criterios de inclusión establecidos (más del 60\% de los cursos de cuarto año aprobados) y 13 por ser encuestas identificadas como «respondidas de manera descuidada o con insuficiente esfuerzo» (en inglés, Careless or Insufficent Effort response, $\mathrm{C} / \mathrm{IE}$ ). La identificación de las respuestas $\mathrm{C} / \mathrm{IE}$ fue realizada bajo algunos de los procedimientos descritos por Curran (2016).

De esta manera, la muestra final está compuesta por 51 estudiantes de cuarto año de las carreras de pedagogía de dos universidades tradicionales de la Región de Antofagasta, de los cuales el 31,37\% (16) son hombres y el 68,63\% (35) son mujeres. E1 21,56\% (11) estudia una pedagogía que está orientada a la atención del nivel inicial de educación, el 43,13\% (22) al nivel básico y medio y el 35,29\% (18) al nivel medio. 
Los cuestionarios fueron aplicados de manera presencial. Antes de la entrega de los instrumentos, los estudiantes leyeron y firmaron un consentimiento informado, en el que se explican los riesgos y resguardos a la privacidad de los datos entregados.

Dicho consentimiento informa que participar del estudio no involucra riesgos para su salud física o mental y que tanto los cuestionarios como la base de datos generada no incluyen ninguna variable que permita identificar personalmente a los estudiantes.

\subsection{Procedimientos y estrategia de análisis}

Con el objetivo de hacer comparaciones, para cada individuo se calculó puntajes en cada escala y sus respectivas dimensiones. Estos puntajes se obtuvieron sumando las respuestas de cada reactivo (escala de 0 a 4) y dividiendo el resultado por el número de ítems. De esta manera, se generaron variables cuyo mínimo es cero y cuyo máximo es cuatro, siendo cero la ausencia del rasgo medido. Por la naturaleza de las variables, la mayoría de ellas no pueden asumirse como continuas, por lo que se procedió a realizar procedimientos no paramétricos.

Se realizaron procedimientos estadísticos descriptivos, tales como cálculo de medidas de tendencia central y cálculo de coeficientes de correlación lineal de Spearman.

Además, se realizaron procedimientos no paramétricos de inferencia estadística como la prueba de Kruskal Wallis y/o la prueba de Mann-Whitney. Para determinar significancia estadística en las relaciones entre variables, se utilizó la prueba para el coeficiente de correlación lineal de Spearman.

Para todas las pruebas estadísticas, fue utilizado un nivel de significancia de 0,05.

Para los procedimientos estadísticos, fueron utilizados los programas ofimáticos Microsoft Excel 2016 y LibreOffice Calc 5, además de los programas estadísticos IBM SPSS Statistics 21 e IBM SPSS Amos 21.

\section{Resultados}

Cuando se comparan los puntajes de la escala de "Actitud hacia la 
Diversidad" entre ambos sexos, se visualiza a las mujeres como aquellas que tienden a poseer una mayor actitud hacia la diversidad personal que los hombres (ver tabla 1 ). Dicha diferencia es estadísticamente significativa $(\mathrm{p}<0,05)$.

Tabla 1. Medias y medianas según sexo en la Escala de Actitud hacia la Diversidad

\begin{tabular}{|c|c|c|c|c|c|}
\hline \multirow[b]{2}{*}{ Dimensión } & \multicolumn{2}{|c|}{ Media } & \multicolumn{2}{|c|}{ Mediana } & \\
\hline & Masculino & Femenino & Masculino & Femenino & \\
\hline Cognitivo & 3,45 & 3,41 & 3,50 & 3,50 & \\
\hline Personal & 2,33 & 2,69 & 2,33 & 2,67 & $*$ \\
\hline Cultural & 3,29 & 3,37 & 3,40 & 3,40 & \\
\hline Escala completa & 3,03 & 3,17 & 3,03 & 3,20 & \\
\hline
\end{tabular}

Nota: las filas marcadas con asterisco indican diferencia significativa en la distribución de los puntajes de cada categoría; se realizó la prueba de Mann-Whitney.

${ }^{*} \mathrm{p}<0,05$

Los resultados expuestos en la tabla 2 manifiestan la presencia de una mayor actitud hacia la diversidad entre aquellos estudiantes que han participado en alguna capacitación o actividad que promueva la inclusión. La diferencia se produce tanto en la escala completa como también en la dimensión "Diversidad Personal", donde se observa que los estudiantes que han participado en actividades inclusivas poseen puntajes más altos respecto a quienes no lo han hecho.

Tabla 2. Medias y medianas según participación en actividades inclusivas en la Escala de Actitud hacia la Diversidad

\begin{tabular}{cccccc}
\hline & \multicolumn{2}{c}{ Media } & \multicolumn{3}{c}{ Mediana } \\
Dimensión & Sí & No & Sí & No & \\
\hline Cognitivo & 3,50 & 3,32 & 3,50 & 3,50 & \\
Personal & 2,72 & 2,39 & 2,67 & 2,33 & $*$ \\
Cultural & 3,44 & 3,22 & 3,40 & 3,25 & \\
Escala completa & 3,24 & 2,99 & 3,30 & 2,98 & $*$ \\
\hline
\end{tabular}


Nota: Las filas marcadas con asterisco indican diferencia significativa en la distribución de los puntajes de cada categoría; se realizó la prueba de Mann-Whitney. Sí=Sí ha participado en actividades inclusivas. $\mathrm{No}=\mathrm{No}_{\mathrm{o}}$ ha participado en actividades inclusivas. ${ }^{*} \mathrm{p}<0,05$. $^{* *} \mathrm{p}<0,01$

Ahora, cuando se contrastan los puntajes de cada dimensión, segmentando la muestra según el nivel educativo al que está orientado la carrera que estudia, nuevamente existen diferencias significativas en la escala completa. Esta diferencia está motivada por los puntajes de la dimensión de "Diversidad Personal". Los resultados apuntan a que los estudiantes que pertenecen a una carrera orientada hacia la educación inicial tienden a poseer una mayor actitud hacia la diversidad. (Ver tabla 3).

Tabla 3. Medias y medianas según tipo de carrera en la Escala de Actitud hacia la Diversidad

\begin{tabular}{cccccccc}
\hline & \multicolumn{3}{c}{ Media } & \multicolumn{3}{c}{ Mediana } \\
& EI & BM & EM & EI & BM & EM & \\
\hline Cognitivo & 3,66 & 3,32 & 3,40 & 4,00 & 3,25 & 3,50 & \\
Personal & 3,11 & 2,49 & 2,36 & 3,17 & 2,42 & 2,33 & $* * *$ \\
Cultural & 3,56 & 3,27 & 3,31 & 3,60 & 3,40 & 3,30 & \\
Escala completa & 3,44 & 3,05 & 3,04 & 3,45 & 3,10 & 2,98 & $* * *$ \\
\hline
\end{tabular}

Nota: Las filas marcadas con asterisco indican diferencia significativa en la distribución de los puntajes de cada categoría; se realizó la prueba de Kruskal-Wallis. EI: Educación inicial. BM: Educación básica y media. EM: Educación media.

**** $\mathrm{p}<0,001$

No se encontraron otras diferencias significativas desde un punto de vista estadístico al observar los puntajes de la Escala de Actitud a la Diversidad con las otras variables.

Ahora bien, cuando se mide la "Inteligencia Emocional" y se contrasta según el sexo de los participantes, es posible observar que los hombres poseen una mayor comprensión emocional que las mujeres $(\mathrm{p}>0,01)$ (ver tabla 4$)$. 
Tabla 4. Medias y medianas según sexo en la Escala de Inteligencia Emocional

\begin{tabular}{cccccc}
\hline & \multicolumn{2}{c}{ Media } & \multicolumn{2}{c}{ Mediana } \\
Dimensión & Masculino & Femenino & Masculino & Femenino & \\
\hline Percepción & 2,47 & 2,76 & 2,50 & 2,88 & \\
Comprensión & 3,18 & 2,62 & 3,19 & 2,63 & $* * *$ \\
Regulación & 2,77 & 2,70 & 2,69 & 2,63 & \\
Escala completa & 2,81 & 2,69 & 2,96 & 2,71 \\
\hline
\end{tabular}

Nota: Las filas marcadas con asterisco indican diferencia significativa en la distribución de los puntajes de cada categoría; se realizó la prueba de Mann-Whitney.

** $\mathrm{p}<0,01$

Por otra parte, cuando se comparan los resultados respecto de la participación en capacitaciones o actividades que promueven la inclusión, se observa que existe un mayor desarrollo de la "Inteligencia Emocional" entre aquellos estudiantes que han participado de dichas actividades (ver tabla 5). Cabe destacar que estas diferencias son significativas en la escala global $(\mathrm{p}<0,05)$ y en la dimensión "Regulación Emocional" $(\mathrm{p}<0,01)$.

Tabla 5. Medias y medianas según participación en actividades inclusivas en la Escala Inteligencia Emocional

\begin{tabular}{cccccc}
\hline & \multicolumn{2}{c}{ Media } & \multicolumn{3}{c}{ Mediana } \\
Dimensión & Sí & No & Sí & No & \\
\hline Percepción & 2,77 & 2,53 & 2,88 & 2,75 & \\
Comprensión & 2,92 & 2,63 & 2,88 & 2,75 & \\
Regulación & 2,94 & 2,44 & 3,13 & 2,25 & $* *$ \\
Escala completa & 2,88 & 2,53 & 3,00 & 2,42 & $*$ \\
\hline
\end{tabular}

Nota: Las filas marcadas con asterisco indican diferencia significativa en la distribución de los puntajes de cada categoría; se realizó la prueba de Mann-Whitney. Sí=Sí ha participado en actividades inclusivas. $\mathrm{No}=\mathrm{No}$ ha participado en actividades inclusivas. ${ }^{*} \mathrm{p}<0,05 .{ }^{* *} \mathrm{p}<0,01$ 
No se encontraron otras diferencias significativas desde un punto de vista estadístico al observar los puntajes de la Escala de Inteligencia Emocional con las otras variables.

Ahora bien, respecto de la relación entre las distintas variables, es posible observar que no existe una relación entre la "Actitud hacia la Diversidad" y la "Inteligencia Emocional" ( $r h o=0.204 ; p=0,152$ ). No obstante, existen correlaciones significativas entre algunas dimensiones de la misma escala (ver tabla 6).

Un aspecto relevante que destacar es la relación significativa entre la dimensión "Regulación Emocional" de la escala "Inteligencia Emocional" y el puntaje global de la escala de "Actitud hacia la Diversidad" (rho $=0,284 ; p=0,043)$, así como también la relación entre esta dimensión y el área de "Diversidad Personal" (rho = 0,279; $p=0,047)$.

Tabla 6. Coeficiente de correlación lineal de Spearman entre dimensiones y escalas

\begin{tabular}{lrrrrrrrr}
\hline & EAD & Cogn & Pers & Cult & EIE & Perc & Comp & Regu \\
\hline EAD & 1,00 & $* * * 0,50$ & $* * * 0,81$ & $* * * 0,75$ & 0,20 & 0,06 & 0,11 & $* 0,28$ \\
Cogn & $* * * * 0,50$ & 1,00 & 0,26 & 0,11 & 0,09 & $-0,25$ & 0,16 & 0,20 \\
Pers & $* * * 0,81$ & 0,26 & 1,00 & $* * 0,39$ & 0,17 & 0,08 & 0,01 & $* 0,28$ \\
Cult & $* * * 0,75$ & 0,11 & $* * 0,39$ & 1,00 & 0,20 & 0,21 & 0,14 & 0,19 \\
EIE & 0,20 & 0,09 & 0,17 & 0,20 & 1,00 & $* * * 0,63$ & $* * * 0,81$ & $* * * 0,86$ \\
Perc & 0,06 & $-0,25$ & 0,08 & 0,21 & $* * * 0,63$ & 1,00 & 0,26 & $* * 0,37$ \\
Comp & 0,11 & 0,16 & 0,01 & 0,14 & $* * * 0,81$ & 0,26 & 1,00 & $* * * 0,58$ \\
Regu & $* 0,28$ & 0,20 & $* 0,28$ & 0,19 & $* * * 0,86$ & $* * 0,37$ & $* * * 0,58$ & 1,00 \\
\hline
\end{tabular}

Nota: Los coeficientes marcados con asterisco indican correlaciones significativas; se realizó la prueba para el coeficiente de correlación lineal de Spearman. EAD=Escala de Actitud hacia la diversidad, Cogn=Diversidad cognitiva, Pers=Diversidad personal, Cult=Diversidad cultural, EIE=Escala de Inteligencia emocional, Perc=Percepción, Comp=Comprensión, Regu=Regulación.

${ }^{*} \mathrm{p}<0,05$. $^{* *} \mathrm{p}<0,01$. $^{* * *} \mathrm{p}<0,001$ 


\section{Discusión y Conclusiones}

Los datos y análisis realizados muestran que, en términos generales, existe una actitud favorable hacia la diversidad personal, cognitiva y cultural y apropiados niveles de inteligencia emocional en los y las estudiantes que estudian pedagogía en dos universidades de la Ciudad de Antofagasta. Dichas variables no solo son importantes para promover espacios inclusivos en el sistema educacional, sino también para mejorar y fortalecer el proceso de enseñanza y aprendizaje de los y las estudiantes (Pérez y Filella, 2019).

Los y las estudiantes que estudian pedagogía en dos universidades pertenecientes al Consejo de Rectores de la Ciudad de Antofagasta declaran en general una actitud favorable hacia la diversidad y niveles adecuados de inteligencia emocional.

Los datos observados muestran que no existe relación entre las "Actitudes hacia la Diversidad" y la "Inteligencia Emocional", no obstante, se encontraron evidencias de una relación significativa entre la dimensión "Diversidad Personal" de la escala Actitudes hacia la diversidad y la dimensión "Regulación Emocional" de la escala Inteligencia Emocional; lo anterior debido probablemente a que la capacidad para regular emociones positivas y negativas de manera eficaz también permite el reconocimiento del otro en cuanto sus características y derechos como individuos (Crespo, 2018).

En relación con las distintas variables que influyen en la Actitud hacia la Diversidad, los hallazgos indican que son las mujeres las que presentan una actitud más favorable en comparación a los hombres, así también los estudiantes que provienen de establecimientos educacionales municipales y aquellos estudiantes que han tenido la posibilidad de participar en algún tipo de actividad que promueva la inclusión. En este sentido, y cuando la diversidad está normalizada, las "actitudes de prejuicio" en sus distintos componentes (cognitivo, afectivo y conductual) tienden a disminuir, lo que podría explicar la actitud favorable hacia la diversidad en estudiantes que provienen tanto de establecimientos municipales y también de aquellos que han participado de actividades pensadas en favorecer la construcción de espacios inclusivos (Díaz, 2002). 
En cuanto a la Inteligencia Emocional, los datos muestran que son los hombres los que presentan una mayor capacidad para percibir, entender, administrar y regular las emociones, así también los y las estudiantes que han tenido la posibilidad de participar en algún tipo de actividad que promueva la inclusión. No obstante, en cuanto a las variables "establecimiento de procedencia" y "nivel educativo de los padres" no se encontraron datos que indiquen la existencia de alguna influencia en la Inteligencia Emocional.

Por último, los datos encontrados permiten concluir que, si bien en esta investigación no se encontraron evidencias de una relación significativa entre la Inteligencia Emocional y las Actitudes hacia la Diversidad (personal cognitiva y cultural), ambas son variables de interés para la educación superior en general y deben ser incorporados en los programas de estudio en la Formación Inicial Docente, si se quiere avanzar en el desarrollo de competencias pedagógicas que permitan la construcción de espacios inclusivos (Jiménez, Sancho y Sánchez, 2019).

Las limitaciones del estudio se relacionan por un lado con la representatividad de la muestra, ya que participaron estudiantes que estudian pedagogía de solo dos instituciones de educación superior de la región de Antofagasta, segunda región de Chile, que tienen -por sus propias condiciones- características particulares y diferentes al resto del país. Otra de limitaciones es el número reducido de la muestra, esto debido a la altísima mortalidad experimental, por lo que se hace necesario para futuras investigaciones incorporar mayor cantidad de estudiantes y una mayor heterogeneidad de la muestra.

\section{Referencias}

Ajzen, I., y Fishbein, M. (1980). Understanding Attitudes and Predicting Social Behavior. Michigan: Prentice-Hall.

Bisquerra, R. (2011). Educación Emocional: Propuestas para educadores y familia. Bilbao: Desclée De Brouwer, S.A.

Cabello, R., Ruiz-Aranda, D. y Fernández-Berrocal, P. (2010). Docentes emocionalmente inteligentes. Revista Electrónica Interuniversitaria de Formación del Profesorado, 13(1), 41-49. 
Crespo, E. (2018). Un enfoque social sobre las emociones. En J. L. Estramiana, y J. R. Torregrosa, La interacción social (pp. 165-183). Madrid: CIS.

Curran, P. (2016). Methods for the detection of carelessly invalid responses in survey data. Journal of Experimental Social Psychology, 66, 4-19.

Díaz, E. (2002). El factor actitudinal en la atención a la diversidad. Profesorado, 6(1-2), 151-165.

Espinoza, M., Sanhueza, O., Ramírez, N. y Sáez, K. (2015). Validación de constructo y confiabilidad de la escala de inteligencia emocional en estudiantes de enfermería. Latino-Am. Enfermagem, 23(1), 139-147.

Extremera, N., y Fernández-Berrocal, P. (2005). Inteligencia emocional percibida y diferencias individuales en el Meta-Conocimiento de los estados emocionales: Una revisión de los estudios con elTMMS. Ansiedad y Estrés, 11(2), 101-122.

Fernández-Barrocal, P., y Extremera, N. (2005). La inteligencia emocional y la educación de las emociones desde el Modelo de Mayer y Salovey. Revista Interuniversitaria de Formacion del Profesorado, 19(3), 63-93.

Infante, M. (2010). Desafios a la Formación Docente: Inclusión Educativa. Estudios Pedagógicos, 1, 287-297.

Jiménez, D., Sancho, P., y Sánchez, S. (2019). Perfil del futuro docente: nuevos retos en el marco de las EEES. Contextos Educativos, 23, 125-139.

Lohr, S. (2000). Muestreo: Diseño y Análisis. México: Thomson.

Mayer, J., y Salovey, P. (1997). "What is emotional intelligence?”. En P. Salovey, y D. Sluyter, Emotional Development and Emotional Intelligence: Implications for Educators (pp. 3-31). New York: Basic Book.

Palomera, R., Fernández-Berrocal, P., y Brackett, M. (2008). Emotional intelligence as a basic competency in pre-service teacher training: some evidence. Electronic Journal of Research in Educational Psychology, 16(2), 437-454.

Paz Maldonado, E. (2018). La formación del profesorado universitario para la atención a la diversidad en la educación superior. Revista de Investigación Educativa, 9(16), 67-82.

Peña, C., Williamson, G., y González-Fiegehen, L. (2006). El rol del gobierno universitario en la promoción de la equidad y la inclusión en la educación superior. En P. Díaz-Romero, Caminos para la Inclusión en la Educación Superior (pp. 281-320). Santiago de Chile: EQUITAS. 
Pérez, N., y Filella, G. (2019). Educación emocional para el desarrollo de competencias emocionales en niños y adolescentes. Praxis y Saber, 10(24), 23-44.

Ramos, V., Piqueras, J., Martínez, A., y Oblitas, L. (2009). Emoción y Cognición: Implicaciones para el Tratamiento. Terapia Psicológica, 27(2), 227-237.

Reyes, L. (2007). La teoría de acción razonada: implicación para el estudio de las actitudes. INED, 7, 66-77.

Rodríguez, A. (1991). Psicología Social. Mexico: Trillas.

Salovey, P., Mayer, J., Goldman, S., Turvey, C., y Palfai, T. (1995). Emotional attention, clarity, and repair: Exploring emotional intelligence using the Trait Meta-Mood Scale. In J. W. Pennebaker (Ed). Emotion, disclosure, $y$ health (pp.125-154). Washington, DC, US: American Psychological Association.

Sanhueza, S., Granada, M., y Bravo, L. (2012). Actitudes del profesorado de Chile y Costa Rica Hacia la Inclusión Educativa. Cuadernos de Pesquisa, 42(147), 884-899.

Sales, A., Moliner, O., y Sanchiz, M.-L. (2001). Actitudes hacia la atención a la diversidad en la formación inicial del profesorado. Revista Electrónica Interuniversitaria de Formación del Profesorado, 4(2), 1-7.

Schutte, N.S., Malouff, J., Bobik, C., Coston, C., Greeson, C., Jedlika, C., y Wendorf, G. (2001). Emotional Intelligence and interpresonal relations. Journal of Social Psychology, 141, 523-536.

Solórzano, M. (2013). Escala para medir actitud hacia la diversidad. Revista Electrónica Actualidades Investigativas en Educación, 13(1), 1-26. 